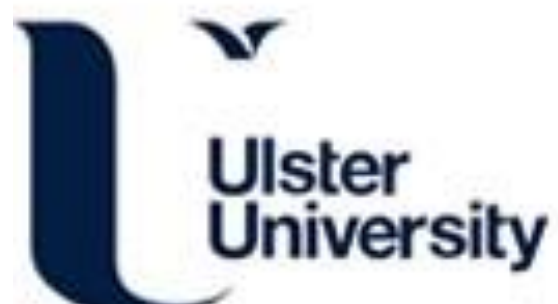

\section{A Computational Model of Neuroreceptor-Dependent Plasticity (NRDP) Based on Spiking Neural Networks}

Espinosa-Ramos , J., Capecci, E., \& Kasabov, N. (2019). A Computational Model of Neuroreceptor-Dependent Plasticity (NRDP) Based on Spiking Neural Networks. IEEE Transactions on Cognitive and Developmental Systems, 11(1), 63-72. https://doi.org/10.1109/TCDS.2017.2776863

Link to publication record in Ulster University Research Portal

Published in:

IEEE Transactions on Cognitive and Developmental Systems

Publication Status:

Published (in print/issue): 01/03/2019

DOI:

10.1109/TCDS.2017.2776863

Document Version

Author Accepted version

\section{General rights}

Copyright for the publications made accessible via Ulster University's Research Portal is retained by the author(s) and / or other copyright owners and it is a condition of accessing these publications that users recognise and abide by the legal requirements associated with these rights.

\section{Take down policy}

The Research Portal is Ulster University's institutional repository that provides access to Ulster's research outputs. Every effort has been made to ensure that content in the Research Portal does not infringe any person's rights, or applicable UK laws. If you discover content in the Research Portal that you believe breaches copyright or violates any law, please contact pure-support@ulster.ac.uk. 


\title{
A Computational Model of Neuroreceptor Dependent Plasticity (NRDP) Based on Spiking Neural Networks
}

\author{
Josafath I. Espinosa-Ramos, Elisa Capecci, and Nikola Kasabov
}

\begin{abstract}
Activity-dependent plasticity has attracted the interest of researchers for years in the domain of computational neuroscience, as the modification of synaptic efficacy occurs as a result of complex biochemical mechanisms that take place at a cellular level. In this paper, we introduce a phenomenological model -implemented as an unsupervised learning rule for spiking neural networks- based on the cross-talk between glutamatergic and GABAergic neuroreceptors: NMDA, AMPA, $\mathbf{G A B A}_{A}$, and $\mathbf{G A B A}_{\mathrm{B}}$. The proposed neuroreceptor-dependent plasticity (NRDP) model is implemented and demonstrated in a spiking neural network environment, NeuCube, for modelling electroencephalography data. We show that the NRDP model can reproduce the generic spike-timing dependent plasticity behaviour in a spiking neural network. In addition, this can be used to simulate changes in excitatory/inhibitory balance in a spiking neural network by altering neuroreceptors activity. More specifically, by varying the parameters that affect neuroreceptors activation, we can study how these changes would affect the learning and memory ability of a subject. In a therapeutic context, this makes it a promising tool for studying the regulatory mechanisms where neuroreceptors cross-talk plays a crucial role. This can lead to new ways of early detection of neurological disorders and for better targeting drug treatments.
\end{abstract}

Index Terms-Artificial neural networks, spiking neural networks, unsupervised learning, synaptic plasticity, activitydependent plasticity, Hebbian rules, neuroreceptors, EEG data, neurological disorders.

\section{INTRODUCTION}

$\mathbf{O}$ VER the last decades, the increase in neurological disorders, especially cognitive impairment and dementia, has created serious health problems with wider social consequences [1]. In response, the scientific community has focused considerable resources and effort on understanding the central nervous system and especially the mechanisms involved in learning and synaptic plasticity, since neurological decline initially affects these functions. New research and better techniques have resulted in a considerable amount of data being made available. However, a new problem has emerged: how to find a suitable technique in order to understand this information and make the best use of it.

In this context, the scientific contribution of information science, especially neuroinformatics, can play a pivotal role. Information science offers new computational techniques based on emulating the cognitive and learning functions of natural

J. I. Espinosa-Ramos*, Elisa Capecci* and Nikola Kasabov* are with the Knowledge Engineering and Discovery Research Institute, Auckland University of Technology, Auckland, 1010 NZ.e-mail: vjier1979@gmail.com, ecapecci@aut.ac.nz,nkasabov@aut.ac.nz (see https://kedri.aut.ac.nz/). intelligent systems such as the human brain. However, many of the techniques already in use are not adequate, not only because the data preparation and filtering steps can take a considerable amount of processing time and cost, but also because they cannot represent the phenomena of study.

As a feasible paradigm, spiking neural networks (SNN) constitute an important approach in neuroscience. They process and communicate information as real neurons do, raising the level of biological realism [2], [3], [4]. Due to their more biologically realistic properties, they can be used to study the operation of neural circuits [5].

SNN models rely on a learning mechanism known as synaptic plasticity for their computational power. Adjusting the synaptic weights can alter the flow of information through a neural network. When the strength of the incoming signal to a neuron is altered, the output signal will also change in strength [6], [7].

A typical approach makes use of Hebbian learning [8], which models neurons' pre- and post- synaptic action potentials transmission such as spike-timing dependent plasticity (STDP) [9]. Variation of STDP as Hebb postulate, have been also proposed [10], [11], [12]. Still, other approaches models biochemical mechanisms at a synaptic connection through either synaptic current modifications (e.g. transmitter-activated ion channels synapses [7] and the synaptic transmission model [13]), or dynamic mechanisms involving non-Hebbian rise and fall terms (e.g. phenomenological model of dynamic synapses [14] and activity-dependent synaptic scaling [15]).

Numerous computational models have been proposed to model the biological basis of activity-dependent plasticity. In [16], synaptic efficacy is modulated by modifying the probability that a vesicle transporting a neurotransmitter is realised depending on the timing of spike in the pre- and post- synaptic neurons. In [17], pre- and post- synaptic signals are modified according to glutamate receptors modulation. Another computational model describing the input current of a neuron as a function of the excitatory and inhibitory conductance of glutamatergic and GABAergic synapses has been proposed in [18]. While, in [19] the author proposed a computational neurogenetic model of a probabilistic spiking neuron that uses three probability parameters and calculates the neuron post-synaptic potential as the contribution of four different types of synapses (fast excitatory, slow excitatory, fast inhibitory and slow inhibitory) that affect the connections between neurons as it was introduced first in [20], [21].

In this paper, we propose a neuroreceptor-dependent plas- 
ticity (NRDP) model utilised as a unsupervised learning in SNN. This is a simplified computational model that emulates the regulation of synaptic plasticity mediated by the cross-talk between glutamate and gaba main neuroreceptors, based on recent evidence in cellular neuroscience [22], [23]. The model was implemented in a brain-like SNN architecture (the NeuCube [24]). This SNN allows to model electroencephalography (EEG) and other brain data, but more importantly, it allows us to study the impact of neuroreceptors on the process of learning of EEG data in the SNN model and to further interpret the findings for the study of brain conditions. This constitute the main contribution given by the NRDP model with respect with already proposed models, as experimental results are not only expressed in terms of classification accuracy, but by visualising the activity and connectivity generated during learning with can study and modulate these changes also by modifying neurorecptors threshold, which could be used to build an in silico simulation that can be used to assist in drug therapy.

As a case study, we used EEG data to classify different patterns and identify functional changes in the neural activity. Spatio temporal brain data (STDB) and especially EEG can provide valuable information for the research and study of cognitive activity in the human cortex as well as the study of cognitive impairment. It has been used extensively for the study of brain functional changes under different conditions, including neurological disease and drug treatments [25], [26], [27]. Additionally, EEG is an affordable technique that can be easily carried around to accommodate patients needs, and it is considered a non-invasive method for the subjects being studied [25].

Together with the NeuCube architecture, the proposed computational model with the use of the NRDP introduces a new set of techniques in the area of neuro- and bio-informatics. This will allow us to better study cognitive brain processes in order to understand how neuroreceptor mediated regulation affects learning and memory processes. By analysing the connectivity and spiking activity generated within this model, the NRDP model could be used as a decision support tool for clinicians to assess treatments and diagnose neurological disorders.

In the next section, we discuss some of the main mechanisms that regulate learning and neural plasticity in the central nervous system as a biological inspiration for the proposed NRDP model. In section III, we describe the dynamics of the NRDP model for its implementation in an SNN. Section IV reports our results and conclusions for the case study of EEG data using the NeuCube methodology. Finally, we discuss a future work on how to extend the methodology and to apply it on other brain data.

\section{AXODENDRITIC CHEMICAL SYNAPSES AND PLASTICITY}

Different kinds of synapses between two neural cells are found in the central nervous system, although axodendritic chemical synapses are the most numerous [28]. Here, the axon of a neural cell (the pre-synaptic cell) enables communication with the other (the post-synaptic cell) through chemical transmissions [29].

At an axon's terminal, molecules called neurotransmitters arrive, carrying stimuli for the target cell; they are released in the synaptic cleft, where they bind to their corresponding receptors located in the dendritic membrane. Depending on the type of neurotransmitter released, they either depolarise the membrane (i.e. excitatory current) and propagate the signal, or hyperpolarise it (i.e. inhibitory current) and suppress the signal [30].

\section{A. Neurotransmitters and Neuroreceptors Involved in the Mechanisms of Learning}

There are many types of neurotransmitters released in a synaptic cleft. The main excitatory neurotransmitter in the central nervous system is glutamate, whilst the main inhibitory neurotransmitter is $\gamma$-aminobutyric acid (GABA) [31]. The most important neuroreceptors activated by glutamate are the $\alpha$-amino-3-hydroxy-5-methyl-4-izoxazole-propionic acid receptors (AMPAR) and the N-methyl-D-aspartate receptor (NMDAR) [32]. The AMPAR is a receptor that mediates fast excitatory synaptic response [33], whilst NMDAR is a ligandgated and voltage-dependent channel related to slow excitatory response [34]. The inhibitory neurotransmitter GABA activates two main classes of neuroreceptors, GABA type $A\left(G_{A B A}\right)$ and GABA type $B\left(G_{A B A}\right)$. They regulate a fast and a slow inhibition of the neural membrane potential respectively [35], [36], [23].

In the hippocampus, complex biochemical mechanisms mediate and process cognitive activities such as learning and memory through the regulation of synaptic plasticity [37]. Several of them occur in both pre- and post-synaptic cells [37]. Two phenomena involved in this process are known as long-term potentiation (LTP) and long-term depression (LTD). Memory formation and modelling synaptic plasticity in the mammalian brain is broadly thought to depend on this bidirectional activity [38], [39]. LTP is responsible for the transfer of information from short-term memory to long-term memory by means of long and persistent depolarisation of the post-synaptic cell's membrane (i.e. repetitive firing stimuli). This mechanism is able to increase synaptic transmission efficiency [40] and was studied by Hebb [8], who postulated the theory known today as Hebbian learning.

These cognitive mechanisms involve the release of a high number of glutamate neurotransmitters by the pre-synaptic cell, which in turn provokes the post-synaptic cell to activate enough receptors and increase their synthesis according to the demand [37]. There, the NMDAR allows the influx of a significant quantity of stimulatory $\mathrm{Ca}^{2+}$ into the cytoplasm [41]. In fact, the influx of these cations is believed to start LTP induction by generating a series of cascade events that are responsible for altering synaptic strength [39]. The NMDAR is a slow excitation neuroreceptor and its plasticity depends mainly on the fast excitation of the AMPAR [37]. In more details, glutamate neurotransmitters in the synaptic cleft bind to both the NMDAR and the AMPAR causing the opening of the latter channel. Subsequently, the influx of $\mathrm{Na}+$ in 
the post-synaptic membrane causes its depolarisation. If the depolarisation event is strong enough, it will provoke the removal of the $\mathrm{Mg}^{2+}$ ion, which obstructs the NMDAR voltage-dependent channel, enabling $\mathrm{Na}^{+}$and $\mathrm{Ca}^{2+}$ to enter the cell [39]. For this reason, NMDAR is a slow excitation receptor and its plasticity, which determines LTP formation, depends on these properties.

In a single synapse, different types of receptors can be found [7], [28]. In hippocampal neurons, NMDAR is broadly found to coexist with $\mathrm{GABA}_{A}$ [22]. However, $\mathrm{GABA}_{\mathrm{B}}$ can also modulate synaptic plasticity through NMDAR regulation in both pre-synaptic cells, by inhibiting $\mathrm{Ca}^{2+}$ and therefore limiting GABA release, and in post- synaptic cells, by mainly co-operating with $\mathrm{GABA}_{\mathrm{A}}$ in inhibiting NMDAR activation [42], [32], [23]. The localisation of both glutamate and GABA receptors in post-synaptic cells depends on neurotransmitters demand, as they are found to move rapidly from where they are synthesised to where they are required in the membrane according to the level of neurotransmitters released by a particular neural cell [32]. The onset of glutamatergic post-synaptic currents suppresses inhibitory functions of GABA receptors and vice versa, inhibitory currents increase proportionally with excitatory activation of glutamate receptors [43], [44], [36]. The balance of these two forces modulates the period of the synaptic activity [45], [36], [34] and that is why these neuroreceptors are highly correlated and modulated [22], [34]. In the literature this dependency is often defined as "crosstalk" (e.g [22], [23]).

Hippocampal neuron mechanisms can be applied to many other neurons encountered in the brain, as they are considered representative of several types of synapses [32]. Studying hippocampal activity is of high relevance for the understanding of neurological disorders, as this region is also one of the first to be affected by the onset of mild cognitive impairment and AD. One hypothesis focuses on the constant activation of NMDAR in AD. High intracellular $C a^{2+}$ leads to mitochondrial dysfunction [46]. Consequently, the constant activation of this receptor leads to a chronic over-activity with an abnormally high synaptic glutamate level under resting condition, with a correspondingly low amount of neurotransmitters left to be released into synaptic cleft during neural activity [41], leading to cellular dysfunction and neural death over a period of time [46].

The study of neural plasticity and its biochemical counterpart is fundamental to the analysis and understanding of the brain's cognitive activity and consequently the information provided by the STBD collected. In this paper, we use some of the main principles of axodentritic chemical synapses to modulate synaptic plasticity in an SNN by modelling the levels of glutamate and GABA receptors (next subsection). Such model is demonstrated on learning spatio-temporal EEG brain data and for the study of the effect of the neuroreceptors on the SNN connectivity.

\section{The Neuroreceptor Dependent Plasticity MODEL (NRDP)}

The NRDP is a phenomenological model that emulates the correlation between glutamate and GABA receptors, which results in learned connection weights between spiking neurons. This relationship generates a persistent potentiation or depression of the synapse that influences the membrane potential and firing activity of the post-synaptic neuron. Here, we define the equations to calculate the synaptic plasticity between two spiking neurons as a function of AMPAR, NMDAR, GABA and $\mathrm{GABA}_{\mathrm{B}}$ levels.

\section{A. Definitions}

A connection between a pre-synaptic neuron $n_{i}$ and a postsynaptic neuron $n_{j}$ is given by:

$$
C_{i j}= \begin{cases}1, & \text { connection; } \\ 0, & \text { no connection }\end{cases}
$$

We define the firing state $S_{i j}$ of a connection $C_{i j}$ (presynaptic spike) at a time $t$ as:

$$
S_{i j}(t)= \begin{cases}1, & \text { spike } \\ 0, & \text { no spike }\end{cases}
$$

Now, let $M=\{A, N\}$ be the set of variables that emulates the glutamate receptors AMPAR and NMDAR respectively, and let $G=\left\{G_{a}, G_{b}\right\}$ be the set of inhibitory receptors that respectively emulates $\mathrm{GABA}_{\mathrm{A}}$ and $\mathrm{GABA}_{\mathrm{B}}$.

\section{B. Dynamics of the NRDP}

As far as the glutamate receptors are concerned, we made several assumptions to compute their values at a time $t$. If a pre-synaptic neuron emits a spike so that the firing state $S_{i j}(t)=1$ then the level of $A$ is calculated as

$$
A(t)=\min \left(\theta_{A^{+}}, A(t-1)+k_{A}\right)
$$

where $t$ is the elapsed time; and $k_{A}$ is the gaining rate for AMPAR. If $A$ reaches a maximum threshold value defined by $\theta_{A^{+}}$, then it maintains this value during a time window $\Delta_{w}$, even if the pre-synaptic neuron $n_{i}$ has not emitted a spike (i.e. $S_{i j}\left(\Delta_{w}\right)=0$ ). In this case the AMPAR is completely activated. On the other hand, if the firing state $S_{i j}(t)=0$ (no stimulus) persists for longer time than $\Delta_{w}$, i.e. $A=\theta_{A^{+}}$ and $\Delta_{t}>\Delta_{w}$, then the inhibitory receptor will manifest a behaviour given by

$$
A(t)=\max \left(\theta_{A^{-}}, A(t-1)-k_{G}\right)
$$

where $\theta_{A^{-}}$is the minimum level of AMPAR, and $k_{G}=f_{G} \cdot G$ is the gain of GABA receptors over this receptor. Here, $f_{G}$ is the rate that a GABA receptor level $G$ affects $A$. This implies a decay on the synaptic weight due to an insufficient stimuli, and therefore simulating the phenomenon of LTD which represents competition between excitatory and inhibitory receptors.

Regarding the NMDAR, it is known that its activation often depends on the AMPAR, especially in mechanisms that involve memory formation in the hippocampus [32], [33], [34], [47]. Here, we propose a simplified model where the NMDAR is activated after the AMPAR reaches its maximum level. If the 
firing state $S_{i j}(t)=1$ and the level of AMPAR is $A=\theta_{A^{+}}$ then:

$$
N(t)=\min \left(\theta_{N^{+}}, N(t-1)+k_{N}\right)
$$

otherwise:

$$
N(t)=\max \left(\theta_{N^{-}}, N(t-1)-k_{N}\right)
$$

where $\theta_{N^{ \pm}}$refers to the maximum and minimum levels of NMDAR; and $k_{N}$ to the gaining rate for NMDAR.

Taking into consideration, that LTP formation is determined by the activity of NMDAR and that NMDAR in hippocampal neurons is broadly found to coexist with $\mathrm{GABA}_{\mathrm{A}}$ and also modulated by $\mathrm{GABA}_{\mathrm{B}}$ [22], [42], [32], [23], we implemented this as a higher probability for $\mathrm{GABA}_{\mathrm{A}}$ and lower probability for $\mathrm{GABA}_{B}$ to regulate its activity. Then, in our model fast and slow inhibition is described by

$$
P_{G_{b}}<P_{G_{a}}, P_{G_{b}}=1-P_{G_{a}}
$$

where $P_{G_{a}}$ indicates the $\mathrm{GABA}_{\mathrm{A}}$ activation probability. Then, the activation function of the inhibitory level of a $G$ receptor in a synapse $C_{i j}$ is denoted by:

$$
G= \begin{cases}G_{a}, & \alpha<P_{G_{a}} \\ G_{b}, & \text { otherwise }\end{cases}
$$

where $\alpha=$ unif $(0,1)$ is a dimensionless random number with a uniform distribution.

The level of a GABA receptor activity in time is defined by:

$$
G(t)= \begin{cases}\max \left(\theta_{G^{-}}, G(t-1)-k_{G_{l}}\right), & S_{i j}(t)=1 \\ \min \left(\theta_{G^{+}}, G(t-1)+k_{G_{d}} \Delta_{t}\right), & \text { otherwise }\end{cases}
$$

where $t$ is the training elapsed time, $k_{G_{l}}$ and $k_{G_{d}}$ are phenomenological losing and gaining rates respectively; $\Delta_{t}$ is the elapsed time after $n_{i}$ emitted the last spike to neuron $n_{j} ; \theta_{G^{ \pm}}$ represents the maximum and minimum threshold values for each GABA receptor. For the sake of simplicity, max and min functions define the receptors' boundary levels.

Finally, the synaptic weight $W_{i j}(t)$ from neuron $n_{i}$ to $n_{j}$ at a time $t$ is calculated as

$$
W_{i j}(t)=N(t)+A(t)-G(t)
$$

In a post-synaptic neuron $n_{j}$, the membrane potential $u_{j}(t)$ at time $t$ depends on a stimulating current given by the sum of the synaptic weights from all connections with a firing state $S_{i j}=1$. In the standard way for leaky integrate-and-fire type spiking neurons, this membrane potential is calculated as

$$
u_{j}(t)=u_{j}(t-1)+\sum_{i=1}^{m} W_{i j}(t)
$$

where $m$ is the number of spiking neurons connected to the neuron $n_{j}$. If $n_{j}$ lacks of stimuli (i.e. $\sum_{i=0}^{m} S_{i j}=0$ ) then its membrane potential decreases by a "leak" value. Firing occurs whenever $u_{j}(t)$ reaches a threshold $\theta_{u}$, then the membrane potential is reset to an initial state $u_{r}$. Afterwards, the neuron dynamics are interrupted during an absolute refractory time $\delta_{r}$ and restart the integration at time $t+\delta_{r}$ with the new initial condition $u_{j}(t)=u_{r}$.

The NRDP model evolves the connectivity and spiking activity of the SNNc. Its stability and accuracy depends on the values of its correlated variables which are strongly related to the data of the case study. Therefore, hyperparameter optimisation is needed for better performance of the SNNc, leading a favourable knowledge discovery.

\section{A Case Study of Cognitive EeG Data}

\section{A. EEG Data Collection}

We collected EEG data from 21 healthy volunteers. Ethical approval 13/283 was granted by the Auckland University of Technology Ethics Committee. The subjects were aged between 28 and 50 years. They had all undertaken undergraduate university study as a minimum, had no history of cognitive impairment, and were all right handed. All measurements were taken in a quiet room with the participants seated in a dining chair and using a computer screen. Data was collected following two scenarios (a resting task and a cognitive task) using the Emotiv EPOC (http://emotiv.com/) device, a standard EEG system with 14 feature channel names based on the international 10-20 electrodes location system, which are AF3, F7, F3, FC5, T7, P7, O1, O2, P8, T8, FC6, F4, F8, AF4 plus two references in the P3/P4 location. No filtering was applied to the data, neither on-line nor off-line, and the recordings were made at a sampling rate of $128 \mathrm{~Hz}$ per channel. Data was collected in one session. The length of each session was $30 \mathrm{~s}$ per scenario, chosen in accordance with the duration of the memory task.

The resting task was recorded with eyes closed in order to avoid disturbing artefacts (such as blinking of the eyes) and each subject was asked to avoid thinking or planning thoughts as much as possible.

For the cognitive task, we performed a modified Stenberg's Memory Scanning Test [48]. In this experiment, each subject was asked to sit comfortably and look at a screen. A fixation cross appeared for 3 seconds; after that, a "positive set" of three random letters was displayed for 5 seconds, to give the subjects enough time to memorise them. After a 3 seconds pause, when the screen turned blank, a single "test set" was presented that may, or may not, have been shown previously. Every subject was asked to decide if the test set was included in the positive set or not. The experiment was repeated increasing the number of elements in the positive set (from three to five and finally to seven letters).

For this study, only the last $15 \mathrm{~s}$ of the $30 \mathrm{~s}$ recorded were used to run the experiments in order to eliminate any possible noise related to the initial experimental settings. Also, one data point for every three was selected from the entire EEG sequence, obtaining one sample of 640 data points per 14EEG channels per class and per subject. This information was considered enough for the algorithm to discriminate the resting scenario from the cognitive task. In total, we obtained 21 samples for class 1 ( $\mathrm{C} 1$, resting) and another 21 samples for class 2 ( $\mathrm{C} 2$, memory). 


\section{B. The NeuCube Architecture}

NeuCube is an SNN architecture for mapping, learning and understanding of STBD. It was initially proposed in [24] and then further developed in [49], [50]. This architecture consists of the following functional modules: an input data encoding module, in which input data is encoded into trains of spikes that are then presented to the main module; the 3D spiking neural network cube ( $\mathrm{SNNc}$ ) module, where time and space characteristics of the STBD are captured and learned in an unsupervised mode; and the output module for data classification (or regression) which applies supervised learning in an SNN classifier/regressor.

The process of creating a NeuCube model for the case study EEG data modelling can be summarised as follows:

- Using the input module, the available EEG data is transformed into spike sequences using the threshold-based algorithm (TBR) [50], as this algorithm identifies just differences in consecutive EEG values.

- The spike trains are entered into the SNNc containing 1471 leaky integrate-and-fire (LIF) neurons mapped according to the Talairach template [51]. The input variables (i.e. the 14 EEG channels) are mapped into spatially located spiking neurons in the SNN cube that correspond to the 3D spatial coordinates of the channels according to the 10-20 system. The synaptic connections were initialised using a small- world connectivity. Then we trained the SNNc on the encoded EEG data, using the proposed NRDP as the unsupervised learning algorithm.

- In the output module, we use the deSNN [52] algorithm for supervised learning to classify the activated patterns produced by the SNNc when the EEG data is entered again.

Previous implementations and experiments with the NeuCube architecture used STDP as the learning algorithm for the unsupervised training. Here, we implemented the NRDP as the learning algorithm. We refer to this architecture as NeuCube-NRDP. A functional diagram of the NeuCube-NRDP is depicted in Figure 1.

\section{Parameter Optimisation}

Since the NRDP involves several variables, a fine tuning is necessary for better classification results. As a starting point, we assumed that equivalent model behaviour can be achieved by using the NRDP or the STDP learning rules. Both of them, can automatically balance the synaptic strengths making postsynaptic firing sensitive to pre-synaptic potentials. In order to produce the same behaviour (spike sequences) when using either STDP and NRDP learning rules, we fitted the NRDP parameters by means the differential evolution (DE/rand/1/bin) algorithm [53] on a small recurrent SNN as shown in Figure 2.

The aim was to maximise the spike time coincidences between the spike trains produced by each neuron using the STDP rule with a learning rate $r=0.01$, and then using the NRDP rule proposed with optimised parameters. These parameters are related to the maximum $\theta_{+}$and the gain $k$ values for each neuroreceptor, the probability of $\mathrm{GABA}_{\mathrm{A}}$ to be expressed $P_{G_{a}}$, and the inhibitory rate $f_{G}$. Each neuron on the SNN was a leaky integrate-and-fire neuron model that fires when the membrane potential reaches a threshold value $t h=0.5$, using a leak value $l k=0.02$ and a refractory time $t_{r}=6 \mathrm{~ms}$. Table I shows the optimised NRDP parameters to produce the same spike trains when using the STDP rule.

TABLE I: NRDP parameter settings that leads to same spiking activity as when using the STDP learning rule.

\begin{tabular}{llr}
\hline Neuroreceptor & Parameters & \multicolumn{1}{c}{ Values } \\
\hline AMPAR & $\theta_{A^{+}}$ & 0.901680 \\
& $k_{A}$ & 0.542872 \\
NMDAR & $\theta_{N^{+}}$ & 0.230013 \\
& $k_{N}$ & 0.011660 \\
GABA $_{\mathrm{A}}$ & $\theta_{G_{a}}$ & 0.755415 \\
& $k_{G_{a}}$ & 0.385908 \\
& $P_{G_{a}}$ & 0.7 \\
GABA $_{\mathrm{B}}$ & $\theta_{G_{b}}$ & 0.795471 \\
& $k_{G_{b}}$ & 0.110321 \\
Inhibitory Rate & $f_{G}$ & 0.01 \\
\hline
\end{tabular}

We can conclude that through setting suitable parameters using the NRDP algorithm, we can achieve the same performance as using the STDP algorithm. Additionally, the NeuCube-NRDP can be used to study how the spiking activity of the SNNc is affected by neuroreceptors modulation. This allows to study how changes in neurorecpetors activity can modulate synaptic plasticity during cognitive processes.

In the next experiments, additional parameters of the NeuCube-NRDP were optimised by a grid search algorithm that evaluated the model based on the best classification accuracy result. The resulting best parameters were set as follows: the threshold value for encoding algorithm was set at 22.518; the small-world connectivity probability parameter $p$ was set at 0.075 ; the spiking neurons threshold of firing $\Theta$, the refractory time $r$ and the potential leak rate $l$ of the LIF neuron model were set at $0.306,5 \mathrm{~ms}$ and 0.02 respectively; finally, the variables mod and drift of the deSNN classifier were set at 0.4 and 0.25 respectively.

After the optimisation procedure, we retained the best NeuCube-NRDP model. The initial connectivity of this model was retained and used to perform the final experiments. We validated the results applying the Monte Carlo cross validation after 30 runs using $50 \%$ of the data randomly selected for training and the remaining $50 \%$ for testing. We also performed two more experiments increasing the parameter $\theta_{A^{+}}$(defining the maximum value for the AMPAR) by $10 \%$ and decreasing it by $10 \%$. With the latter experiments, we demonstrated the ability of the NeuCube-NRDP to model the effect of neuroreceptors on the connectivity of the learned SNN model from EEG data, therefore to offer some meaningful interpretations of the model in terms of brain learning and memory functions.

\section{Results and Discussion}

The NeuCube-NRDP architecture allowed us to analyse both the data and the brain-like processes that generated it, including the changes of its functionality across different conditions and groups of subjects. When we modified the 


\section{Input Module}

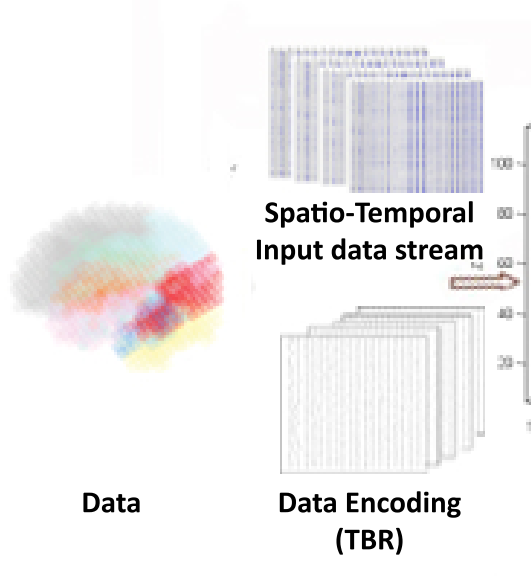

NeuCube Module

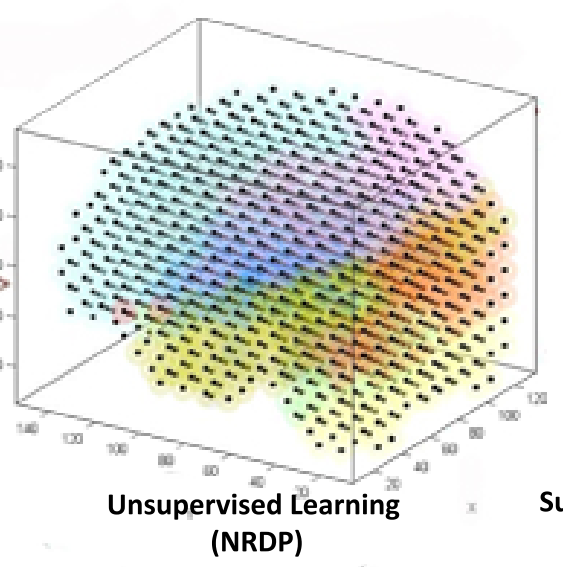

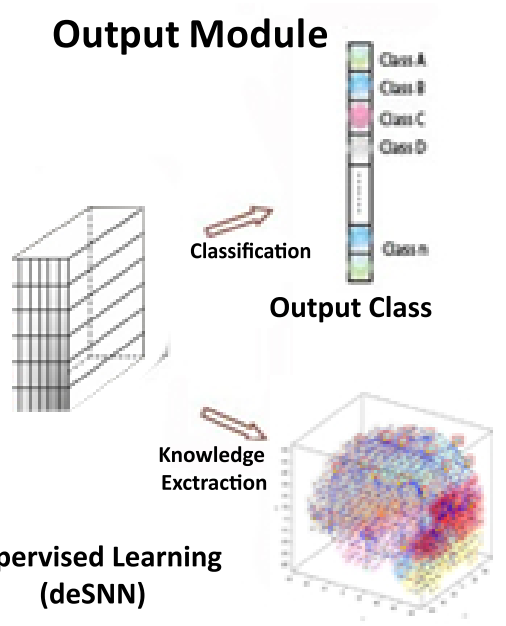

SNNc Analysis

Fig. 1: A general NeuCube-NRDP architecture.
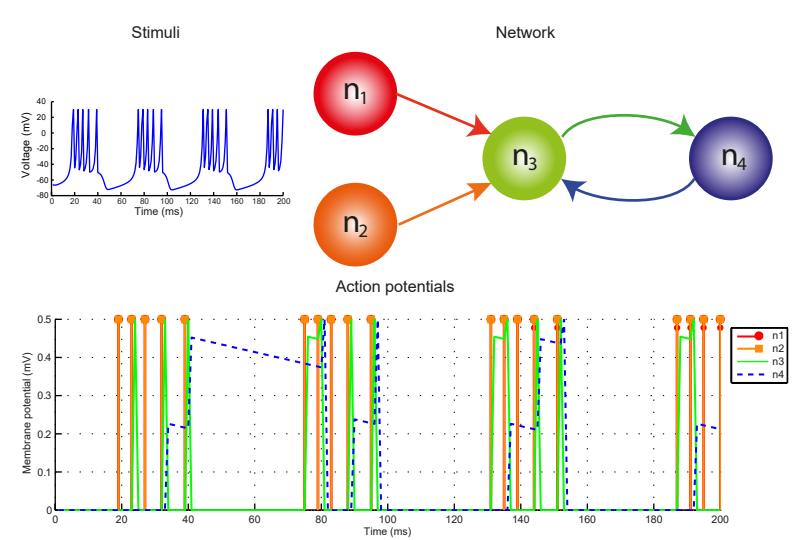

Fig. 2: Spike trains generated after stimulating a recurrent spiking neural network with a periodic burst of spikes, and using the STDP or NRDP learning rules.

level of glutamate and GABA receptors, we could observe how the neuroreceptors affected the spiking activity and the connectivity of the SNNc, and therefore the classification results.

1) Classification: In Table II, we report the classification accuracy results obtained by using the optimised parameter values and the modified $\theta_{A^{+}}$values.

TABLE II: Classification accuracy. The results are presented as classification accuracy percentage for class 1 (resting), class 2 (memory), and overall.

\begin{tabular}{lrrr}
\hline Parameters & Class 1 & Class 2 & Overall \\
\hline Optimised & $91 \%$ & $64 \%$ & $77 \%$ \\
Increase in $\theta_{A^{+}}+10 \%$ & $36 \%$ & $73 \%$ & $55 \%$ \\
Decrease in $\theta_{A^{+}}-10 \%$ & $73 \%$ & $36 \%$ & $55 \%$ \\
\hline
\end{tabular}

We observe that optimised parameter values resulted in a good classification accuracy of $77 \%$ overall. Considering the short training period, the noisy nature of the data, its rather informal collection method, and the technical limitations of the EEG recording device, this result is satisfactory. Thus, the NRDP algorithm demonstrated its ability to properly distinguish between the resting and the memory tasks. However, modifying the maximum value of the AMPAR expression $\theta_{A^{+}}$ parameter affects the classification accuracy by both class and overall. After increasing $\theta_{A^{+}}$value by $10 \%$ the classification accuracy for class 1 (the resting state) considerably decreased. We assume that the increment of the threshold would prevent subjects to get into a resting state. In contrast, decreasing the $\theta_{A^{+}}$value by $10 \%$ makes the samples belonging to class 2 (the memory task) difficult to classify. A simple explanation is that a decrease in the threshold does not support memory activity.

2) Analysis of the Connectivity of the SNNc in NeuCubeNRDP: By visualising the connectivity of the training SNNc models in the experiment above (see Table II), we can study how time-series EEG data is learned during unsupervised training, and to possibly extract new knowledge from it. We aim to understand how spiking activity and connectivity evolve during learning, for each of the two classes. Figure 4-5 shows the learning connectivity in 3D SNNc along with the corresponding 12 brain functional areas. Yellow-green denotes the temporal lobe; pink, the parietal lobe; light-blue, the frontal lobe; red, the fronto- temporal space; light-yellow, the posterior lobe; orange, the occipital lobe; green, the anterior lobe; blue, the sub-lobar region; grey, the limbic lobe; purple, the pons; blue-green, the midbrain; and brown, the medulla. Also, the 14 input neurons are highlighted and labeled in yellow according to their corresponding EEG channels. In figure, blue lines indicate the excitatory synapses and red lines the inhibitory synapses; the thickness of these lines indicates the strength of the activity between neurons.

We observe that modifying the threshold of the maximum value of AMPAR caused the SNNc to evolve different connectivity for both classes across the brain regions.

In Fig. 3, we show the connectivity when the NeuCube was trained with optimised parameter settings. We observe that there is a significant difference between the SNNc con- 

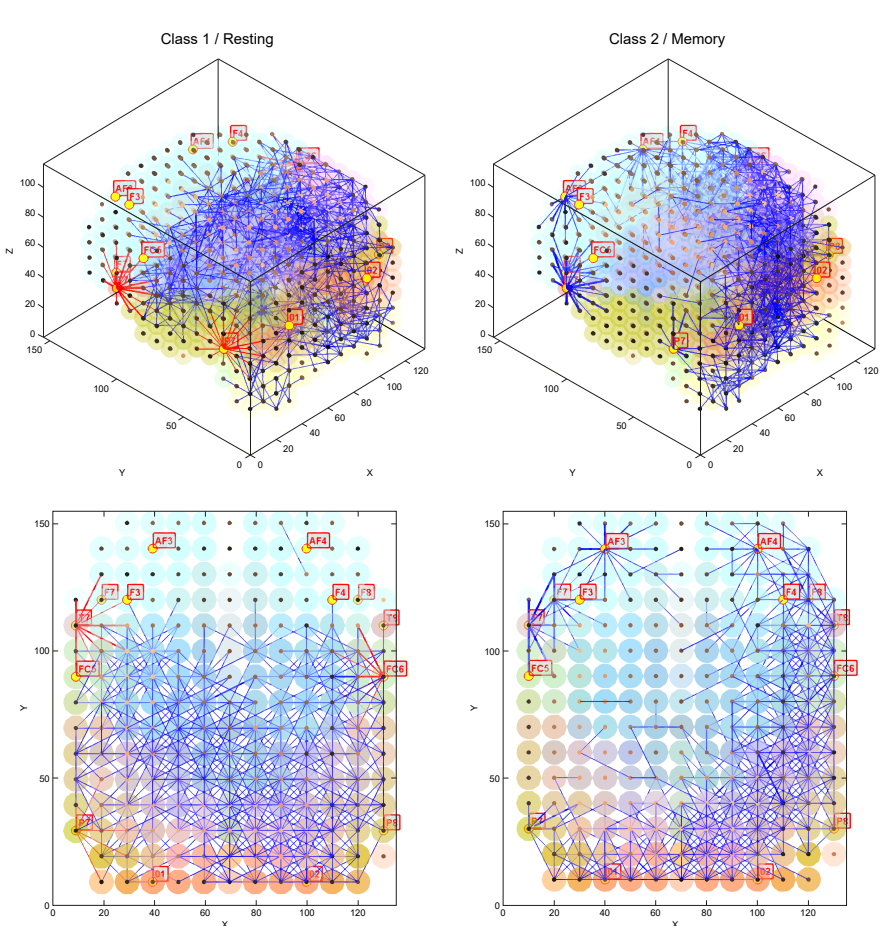

Fig. 3: NeuCube-NRDP connectivity obtained using optimised NRDP parameters. The figure shows the SNNc trained with the EEG data recorded during either the resting task (class 1 ), or the memory task (class 2). The figures show the SNNc connectivity, both in a 3D and in a 2D view, together with the corresponding 12 brain functional areas. Connections in blue color are excitatory and in red - inhibitory.

nectivity produced by the two groups of subjects. The neural activity recorded during the resting task generated an equally distributed connectivity over the entire brain. Contrarily, the memory task evolved most of the connections in the (right) occipito-parietal cortex and centro-temporal area. These findings are consistent with those reported in the literature, as the parietal-temporal-occipital (PTO) area of the human brain is linked with the associative type of memory related to perception and execution processes [54].

Analysing the connectivity of the evolved NeuCube models may lead to new findings about the data and the brain processes that generated it. Each connection in the SNNc represents a spatio-temporal causal relationship between the connected neurons that represent corresponding brain areas. When we increased the AMPAR threshold by $10 \%$ of its initial value (Figure 4), we observed that the evolved connectivity in the SNNc differed between the two groups. Inhibitory connections increased for both classes. The resting task data generated increased connectivity in the temporal area of the left hemisphere, whilst the other areas decreased their connectivity. The memory task data showed increased inhibitory connections in the occipital area (especially by the electrodes $\mathrm{O} 1$ and $\mathrm{O} 2$ ), while excitatory connections increased in the right fronto-temporal area. In fact, over-expression of AMPAR was responsible for affecting the resting state data, as it increased the connectivity, making the classification task more difficult.
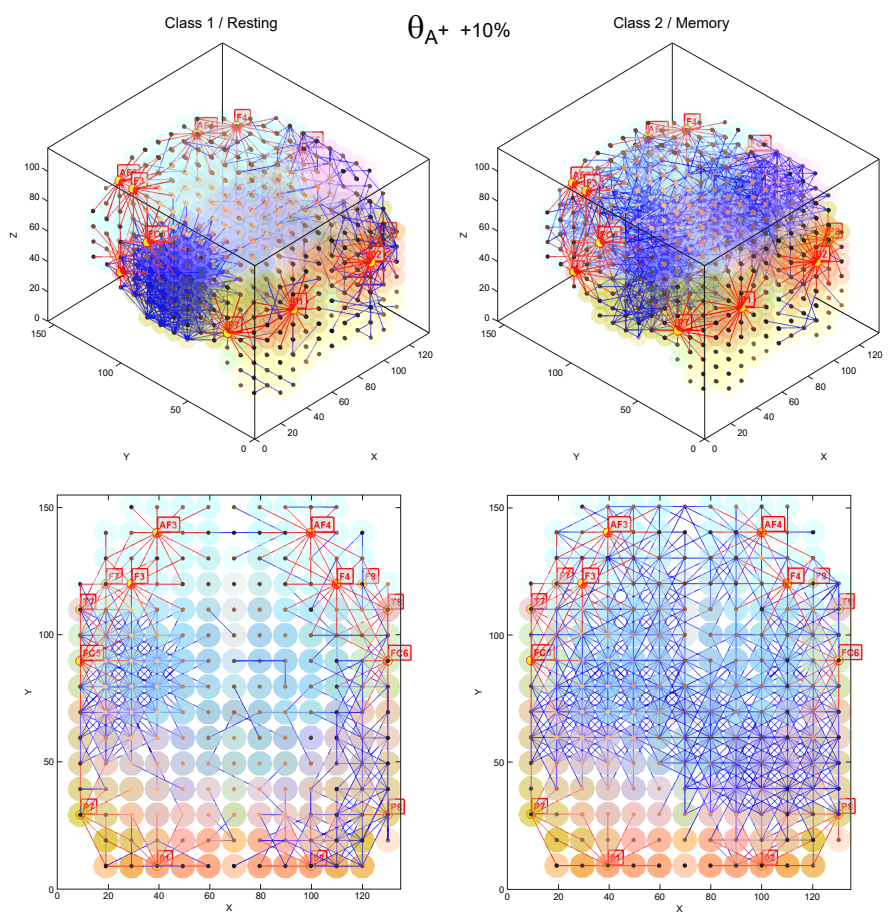

Fig. 4: NeuCube-NRDP connectivity obtained using optimised NRDP parameters with $10 \%$ higher AMPAR activation threshold $\theta_{A^{+}}$. The figure shows the SNNc trained with the EEG data recorded during either the resting task (class 1), or the memory task (class 2). The figures show the SNNc connectivity, both in a $3 \mathrm{D}$ and in a $2 \mathrm{D}$ view, together with the corresponding 12 brain functional areas. Connections in blue color are excitatory and in red - inhibitory.

On the other hand, decreasing the AMPAR threshold by $10 \%$ of its initial value (Fig. 5), resulted in increased inhibitory connections around the input neurons in the resting task data; and for excitatory connections to be generated by the fronto-temporal (right) area. The memory task data generated remarkably increased inhibitory connections in the occipital area. Thus, it is the increased activity of GABAergic synapses that affects the classification results; as inhibitory activity prevails over excitatory affecting the normal neural equilibrium. However, cognitive functions and neural synchronisation are also mediated by GABA transmission, which is important for the correct evolution of the neural process [55].

\section{CONCLUSion ANd Future Work}

In this study, computational modelling of EEG data was achieved by implementing a proposed NRDP learning rule in the NeuCube architecture. Using this system, EEG and other brain data could be used to study the impact of neuroreceptor activity on the post-synaptic potential of the neurons that form the SNNc. Importantly, the evolved connectivity and spiking activity learned could be further interpreted and new knowledge about functional brain conditions could be revealed.

In addition, future research could cover:

- Apply the extended model to test its ability to predict not only between resting and memory states, but also subjects 

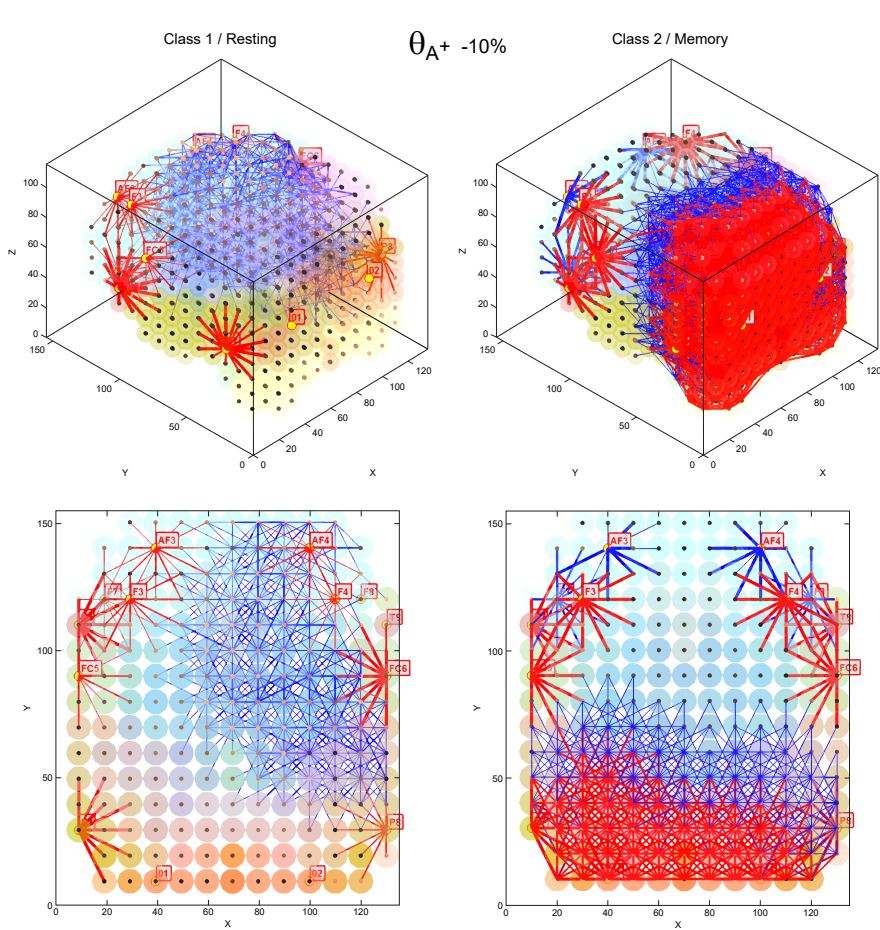

Fig. 5: NeuCube-NRDP connectivity using optimised NRDP parameters with $10 \%$ lower AMPAR activation threshold $\theta_{A^{+}}$. The figure shows the SNNc trained with the EEG data recorded during either the resting task (class 1), or the memory task (class 2). The figures show the SNNc connectivity, both in a $3 \mathrm{D}$ and in a $2 \mathrm{D}$ view, together with the corresponding 12 brain functional areas. Connections in blue color are excitatory and in red - inhibitory.

correct or incorrect response in the Stenbergs memory Scanning Test;

- conduct more experiments using data sets related to the progression of neurodegenerative brain diseases, such as $\mathrm{AD}$ or vascular dementia;

- Extension of the proposed methodology with the introduction of the probabilistic model of a neuron as proposed in [19];

- Extension of the proposed methodology with a gene regulatory network (GRN) module to study how gene expression data can be used to modify the SNNc neural activity (as explained below);

The expression of certain genes triggers mechanisms that are fundamental for the activities of the brain. When a gene is turned on in a neural cell, this is the starting engine of the various brain processes that determine its functions and development. Proteins influence the spiking activity of neural cells, which consequently is reflected in the collected STBD. Genetic parameters are extremely useful especially if we aim to develop a valid neurological computational model. To construct a biologically plausible computational model with the use of SNN, the inclusion of a GRN sub-model is of pivotal importance.

This approach has been taken by several researchers, who have demonstrated that modelling even just a single gene [20] or constructing a GRN [20] contributes to the optimisation of the results and to a better understanding of the phenomena of study. In [20] and [19] a neurogenetic model of a neuron is proposed and studied. The model utilises information about how the main neuroreceptors, and the genes that affect their expression related to the spiking activity of a neuron in terms of fast excitation, fast inhibition, slow excitation and slow inhibition. This model can be also optimised using a gene/protein regulatory network, controlling the dynamic interactions between genes and proteins over time, which affect the post-synaptic potential of the neurons in the SNNc.

The NeuCube-NRDP model proposed here could be extended by adding genetic information, related to NMDAR in particular, in terms of a GRN. NMDAR plays a pivotal role in memory plasticity [38], [39] and it is well known that people affected by AD suffer from cognitive decline and disorientation as first symptoms [39]. In the human brain, the information transmitted by neurons is processed and consolidated in the hippocampus from short-term memory to long-term memory; hence this is one of the first regions to suffer damage as $\mathrm{AD}$ advances. The human brain has two hippocampi, which are part of the limbic system and located in the medial temporal lobes. One of the processes associated with the development of $\mathrm{AD}$ is the constant activation of the NMDAR. This event is thought to lead to an abnormal over-activity during neural processes [41] leading to cellular dysfunction and neural death over a period of time [46]. Moreover, these processes and related mechanisms are known to contribute to the effects of amyloid-beta, which is the major component of senile plaques, one of the typical features of $\mathrm{AD}$ formation and degeneration [56], [32], [41].

It is important to outline that NMDAR is a channel with a quaternary-structure (i.e. a three-dimensional or spatial structure of different proteins). Each of these proteins is encoded by a different gene [57]. The synthesis of this neuroreceptor is only possible due to the simultaneous expression of these genes, as they are responsible for each unit that forms the macromolecule. To construct a GRN for the NMDAR, genes GRIN1, GRIN2A, GRIN2B, GRIN3A and GRIN3B could be used; since, in humans, the NMDAR principal sub-unit is NR1 (encoded by GRIN1 gene) [58]; NR2A and NR2B (encoded by GRIN2A and GRIN2B gene respectively) are found in glutamatergic synapses [32]; NR3A (encoded by GRIN3A gene) has been found in embryonic brain tissue and together with NR3B (gene GRIN3B) it has an inhibitory influence in NMDAR expression [59].

The NRDP model proposed here, implemented in the NeuCube architecture, constitutes only a preliminary step towards further development of new neurogenetic computational models, representing more closely biological neural networks in order to allow for a better understanding of brain cognition.

\section{ACKNOWLEDGMENT}

The study presented in this paper was supported by the National Council of Science and Technology of Mexico (CONACYT) under Grant 348463, and the SRIF INTEL- 
LECTE project of the Knowledge Engineering and Discovery Research Institute (KEDRI, http://www.kedri.info), funded by the Auckland University of Technology. Several people have contributed to the research that resulted in this paper, especially: Y.Chen, J.Hu, E.Tu, L.Zhou, M.Gholami. Many thanks also to Diana Kassabova, Anne French, James C. Veale and Bruce Kerr for proof-reading the text. A limited trial version of the NeuCube software environment is available free for research and teaching from the KEDRI web site https://kedri.aut.ac.nz/R-and-D-Systems/neucube.

\section{REFERENCES}

[1] C. Pritchard, A. Mayers, and D. Baldwin, "Changing patterns of neurological mortality in the 10 major developed countries - 1979 2010," Public Health, vol. 127, no. 4, pp. 357 - 368, 2013.

[2] W. Maass, "Networks of spiking neurons: the third generation of neural network models," Neural networks, vol. 10, no. 9, pp. 1659-1671, 1997.

[3] W. Gerstner, Plausible Neural Networks for Biological Modelling, H. Mastebroek and H. Vos, Eds. Dordrecht: Kluwer Academic Publishers, 2001, vol. What's different with spiking neurons?

[4] E. M. Izhikevich, "Polychronization: Computation with spikes," Neural Computation, vol. 18, no. 2, pp. 245-282, February 2006.

[5] J. Reggia, E. Ruppin, and D. Glanzman, Disorder of Brain, Behavior and Cognition: the Neurocomputational Perspective, ja reggia and e ruppin and dl glanzman ed. Elsevier, 1999.

[6] S. Furber and S. Temple, "Neural systems engineering," Journal of the Royal Society interface, vol. 4, no. 13, pp. 193-206, 2007.

[7] W. Gerstner and W. M. Kistler, Spiking neuron models: Single neurons, populations, plasticity. Cambridge University press, 2002.

[8] D. O. Hebb, The organization of behavior: A neuropsychological approach. John Wiley \& Sons, 1949.

[9] Song, K. D. Miller, and L. F. Abbott, "Competitive hebbian learning through spike-timing-dependent synaptic plasticity," Nature neuroscience, vol. 3, no. 9, pp. 919-926, 2000.

[10] M. Suri, D. Querlioz, O. Bichler, G. Palma, E. Vianello, D. Vuillaume, C. Gamrat, and B. DeSalvo, "Bio-inspired stochastic computing using binary cbram synapses," IEEE Transactions on Electron Devices, vol. 60, no. 7, pp. 2402-2409, 2013.

[11] C. Piochon, P. Kruskal, J. MacLean, and C. Hansel, "Non-hebbian spiketiming-dependent plasticity in cerebellar circuits," Frontiers in Neural Circuits, vol. 6, p. 124, 2013.

[12] A. Tavanaei, T. Masquelier, and A. S. Maida, "Acquisition of visual features through probabilistic spike-timing-dependent plasticity," in Neural Networks (IJCNN), 2016 International Joint Conference on. IEEE, 2016, pp. 307-314.

[13] C. Koch and I. Segev, Methods in Neuronal Modeling: From Ions to Networks, ser. A Bradford book. MIT Press, 1998.

[14] M. V. Tsodyks, K. Pawelzik, and H. Markram, "Neural networks with dynamic synapses," Neural computation, vol. 10, no. 4, pp. 821-835, 1998.

[15] G. G. Turrigiano, K. R. Leslie, N. S. Desai, L. C. Rutherford, and S. B. Nelson, "Activity-dependent scaling of quantal amplitude in neocortical neurons," Nature, vol. 391, no. 6670, pp. 892-896, Feb 1998.

[16] W. Senn, H. Markram, and M. Tsodyks, "An algorithm for modifying neurotransmitter release probability based on pre-and postsynaptic spike timing," Neural Computation, vol. 13, no. 1, pp. 35-67, 2001.

[17] A. Saudargiene, B. Porr, and F. Wrgtter, "How the shape of pre- and postsynaptic signals can influence stdp: A biophysical model," Neural Computation, vol. 16, no. 3, pp. 595-625, March 2004.

[18] J.-N. Teramae and T. Fukai, "Local cortical circuit model inferred from power-law distributed neuronal avalanches," Journal of computational neuroscience, vol. 22, no. 3, pp. 301-312, 2007.

[19] N. Kasabov, "To spike or not to spike: A probabilistic spiking neuron model," Neural Networks, vol. 23, no. 1, pp. 16-19, 2010.

[20] L. Benuskova and N. Kasabov, Computational Neurogenetic Modelling. NY: Springer, 2007.

[21] N. Kasabov, L. Benuskova, and S. G. Wysoski, "Biologically plausible computational neurogenetic models: Modeling the interaction between genes, neurons and neural networks," Journal of Computational and Theoretical Nanoscience, vol. 2, no. 4, pp. 569-573, Dec 2005.

[22] A. N. Shrivastava, A. Triller, and W. Sieghart, "Gabaa receptors: postsynaptic co-localization and cross-talk with other receptors," Frontiers in Cellular Neuroscience, vol. 5, no. 7, p. 97, 2011.
[23] S. Kantamneni, "Cross-talk and regulation between glutamate and gaba(b) receptors," Frontiers in Cellular Neuroscience, vol. 9, no. 135, p. 7, Mar 2015 .

[24] N. Kasabov, "Neucube: A spiking neural network architecture for mapping, learning and understanding of spatio-temporal brain data," Neural Networks, vol. 52, pp. 62-76, 2014.

[25] G. Rodriguez, F. Copello, P. Vitali, G. Perego, and F. Nobili, "Eeg spectral profile to stage alzheimer's disease," Clinical Neurophysiology, vol. 110, pp. 1831-1837, 1999.

[26] S. J. M. Smith, "Eeg in the diagnosis, classification, and management of patients with epilepsy," Journal of Neurology, Neurosurgery \& Psychiatry, vol. 76, no. suppl 2, pp. ii2-ii7, 2005. [Online]. Available:

[27] J. J. Daly and J. R. Wolpaw, "Brain computer interfaces in neurological rehabilitation," The Lancet Neurology, vol. 7, no. 11, pp. 1032 - 1043 , 2008. [Online]. Available:

[28] P. Ernst, Cellular Neuroscience, 1st ed. World Technologies, 2012.

[29] M. Gazzaniga, R. Ivry, and G. Mangun, Cognitive Neuroscience: The Biology of the Mind. W.W. Norton, 2009.

[30] R. Carter, S. Aldridge, M. Page, and S. Parker, The Human Brain Book. DK Publishing, 2009. [Online]. Available:

[31] M. J. T. FitzGerald, G. Gruener, and E. Mtui, Clinical neuroanatomy and neuroscience. Saunders/Elsevier, 2012.

[32] J. I. Collingridge and Y. Wang, "Receptor trafficking and synaptic plasticity," Nature Reviews Neuroscience, vol. 5, pp. 952-962, 2004.

[33] J. Chin, E. Roberson, and L. Mucke, "Molecular aspects of memory dysfunction in alzheimer's disease," in Learning and Memory: A Comprehensive Reference, J. H. Byrne, Ed. Oxford: Academic Press, 2008, vol. 4 , no. 15 , pp. $245-293$.

[34] J. M. Wieronska, K. Stachowicz, G. Nowak, and A. Pilc, "The loss of glutamate-gaba harmony in anxiety disorders," Prof. Vladimir Kalinin (Ed.), no. 8, pp. 135-158, 2011.

[35] R. A. Nicoll, R. C. Malenka, and J. A. Kauer, "Functional comparison of neurotransmitter receptor subtypes in mammalian central nervous system," Physiological reviews, vol. 70, no. 2, pp. 513-565, 1990.

[36] E. O. Mann, M. M. Kohl, and O. Paulsen, "Distinct roles of gabaa and gabab receptors in balancing and terminating persistent cortical activity," The Journal of Neuroscience, vol. 29, no. 23, pp. 7513-7518, 2009.

[37] S. Brady, G. Siegel, R. W. Albers, and D. Price, Basic neurochemistry: principles of molecular, cellular, and medical neurobiology, 8th ed. Academic Press, Nov 2011.

[38] S.-J. Tsai, H.-C. Liu, T.-Y. Liu, C.-Y. Cheng, and H. Chen-Jee, "Association analysis for the genetic variants of the nmda receptor subunit $2 \mathrm{~b}$ and alzheimer's disease," Dementia and geriatric cognitive disorders, vol. 13, no. 2, pp. 91-4, 2002.

[39] S. F. Cooke and T. V. P. Bliss, "Plasticity in the human central nervous system," Brain, vol. 129, no. 7, pp. 1659-1673, 2006. [Online]. Available:

[40] T. Lømo, "The discovery of long-term potentiation." Philosophical Transactions of the Royal Society B: Biological Sciences, vol. 358, no. 1432, pp. 617-620, Apr 2003.

[41] P. T. Francis, "The interplay of neurotransmitters in alzheimer's disease," The International Journal of Neuropsychiatric Medicine, vol. 11, no. 18, pp. 6-9, 2005.

[42] R. W. Olsen and T. M. DeLorey, "Gaba receptor physiology and pharmacology," in Basic Neurochemistry: Molecular, Cellular and Medical Aspects, G. Siegel, B. Agranoff, R. Albers, S. Fisher, and M. Uhler, Eds. Philadelphia: Lippincott-Raven, 1999. [Online]. Available:

[43] Y. Shu, A. Hasenstaub, and D. A. McCormick, "Turning on and off recurrent balanced cortical activity," Nature, vol. 423, no. 6937, pp. 288293, 2003.

[44] B. Haider, A. Duque, A. R. Hasenstaub, and D. A. McCormick, "Neocortical network activity in vivo is generated through a dynamic balance of excitation and inhibition," The Journal of neuroscience, vol. 26, no. 17, pp. 4535-4545, 2006.

[45] A. Compte, M. V. Sanchez-Vives, D. A. McCormick, and X.-J. Wang, "Cellular and network mechanisms of slow oscillatory activity ( $i 1$ $\mathrm{hz}$ ) and wave propagations in a cortical network model," Journal of neurophysiology, vol. 89, no. 5, pp. 2707-2725, 2003.

[46] E. Mohandas, V. Rajmohan, and B. Raghunath, "Neurobiology of alzheimer's disease," Indian Journal of Psychiatry, vol. 51, no. 1, pp. 55-61, 2009. [Online]. Available:

[47] L. Hebert, P. Scherr, J. Bienias, D. Bennett, and D. Evans, "State-specific projections through 2025 of alzheimer disease prevalence," Neurology, vol. 62 , no. 9, pp. $1645-, 2004$. 
[48] W. Van Der Elst, M. P. Van Boxtel, G. J. Van Breukelen, and J. Jolles, "Assessment of information processing in working memory in applied settings: the paper and pencil memory scanning test," PSYCHOLOGICAL MEDICINE-LONDON-, vol. 37, no. 9, p. 1335, 2007.

[49] E. Tu, N. Kasabov, M. Othman, Y. Li, S. Worner, J. Yang, and Z. Jia, "Neucube(st) for spatio-temporal data predictive modelling with a case study on ecological data," in Neural Networks (IJCNN), 2014 International Joint Conference on, July 2014, pp. 638-645.

[50] N. Kasabov, N. M. Scott, E. Tu, S. Marks, N. Sengupta, E. Capecci, M. Othman, M. G. Doborjeh, N. Murli, R. Hartono, J. I. EspinosaRamos, L. Zhou, F. B. Alvi, G. Wang, D. Taylor, V. Feigin, S. Gulyaev, M. Mahmoud, Z.-G. Hou, and J. Yang, "Evolving spatio-temporal data machines based on the neucube neuromorphic framework: Design methodology and selected applications," Neural Networks, pp. -, 2015.

[51] L. Koessler, L. Maillard, A. Benhadid, J. P. Vignal, J. Felblinger, H. Vespignani, and M. Braun, "Automated cortical projection of eeg sensors: anatomical correlation via the international 10-10 system," Neuroimage, vol. 46, no. 1, pp. 64-72, 2009.

[52] N. Kasabov, K. Dhoble, N. Nuntalid, and G. Indiveri, "Dynamic evolving spiking neural networks for on-line spatio- and spectro-temporal pattern recognition," Neural Networks, vol. 41, pp. 188 - 201, 2013.

[53] R. Storn and K. Price, "Differential evolution-a simple and efficient heuristic for global optimization over continuous spaces," Journal of global optimization, vol. 11, no. 4, pp. 341-359, 1997.

[54] J. M. Fuster, "The prefrontal cortex makes the brain a preadaptive system," Proceedings of the IEEE, vol. 102, no. 4, pp. 417-426, 2014.

[55] G. Gonzalez-Burgos, K. N. Fish, and D. A. Lewis, "Gaba neuron alterations, cortical circuit dysfunction and cognitive deficits in schizophrenia," Neural plasticity, vol. 2011, p. 24, 2011.

[56] M. J. Rowan, I. Klyubin, W. K. Cullen, and R. Anwyl, "Synaptic plasticity in animal models of early alzheimer's disease," Philosophical Transactions of the Royal Society of London. Series B: Biological Sciences, vol. 358, no. 1432, pp. 821-828, 2003. [Online]. Available:

[57] T. Ishii, "Molecular charachterization of the family of the n-methyld-aspartate receptor subunits," J. Biol. Chem, vol. 268, p. 2836ñ2843, 1993.

[58] A. Stephenson, "Structure and trafficking of nmda and gabaa receptors," Biochemical Society Transactions, vol. 34, pp. 877-881, 2006.

[59] O. Bendel, B. Meijer, Y. Hurd, and G. von Euler, "Cloning and expression of the human nmda receptor subunit $\mathrm{nr} 3 \mathrm{~b}$ in the adult human hippocampus," Neuroscience Letters, vol. 377, no. 1, pp. 31 - 36, 2005.

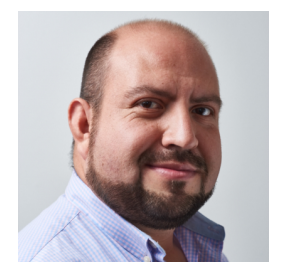

Josafath I. Espinosa-Ramos Dr Josafath Israel Espinosa Ramos holds an MSc in cybernetics from La Salle University, Mexico City, and a PhD in computer science at the Centre for Computing Research of the National Polytechnic Institute, Mexico City. His primary research interests are in the areas of computational neuroscience, evolutionary algorithms, and machine learning. Currently, he works as a Research Fellow for the SRIF 17 INTERACT project at KEDRI. The aim of his research is modelling multi-sensory and multivariate streaming data, and analysing the spatial and temporal relationships among the variables that describe the dynamics of a sensor network.

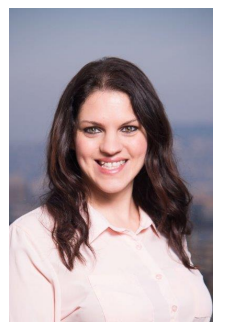

Elisa Capecci Dr Elisa Capecci holds a MSc equivalent in Biochemistry from the University of Granada, Spain, and a PhD in Computer Science from AUT. She works as a Research Fellow for the SRIF 17 INTERACT project at KEDRI and is a lecturer of the Bioinformatics course. She is also investigating the early prediction of cognitive impairment and neurological disorders with spiking neural networks methodology. Her main research interests are in the areas of bioinformatics, computational biology and chemistry, computational neuroscience and machine

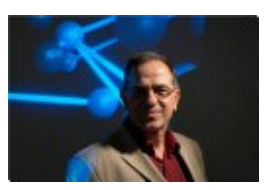

Nikola Kasabov Professor Nikola Kasabov is the Foundation Director of KEDRI, and a Chair of Knowledge Engineering at the School of Computer and Information Sciences at AUT, Fellow of the Royal Society of New Zealand, Fellow of the New Zealand Computer Society and a Fellow of IEEE. $\mathrm{He}$ holds a MSc and PhD from the Technical University of Sofia. His main research interests are in the areas of: intelligent information systems, soft computing, neuro-computing, bioinformatics, brain knowledge discovery.

learning. 